\title{
Superconducting linac at Inter-University Accelerator Centre: Operational challenges and solutions
}

\author{
S. Ghosh, R. Mehta, G. K. Chowdhury, A. Rai, P. Patra, B. K. Sahu, A. Pandey, D. S. Mathuria, J. Chacko, A. Chowdhury, \\ S. Kar, S. Babu, M. Kumar, S. S. K. Sonti, K. K. Mistry, J. Zacharias, P. N. Prakash, T. S. Datta, A. Mandal, \\ D. Kanjilal, and A. Roy \\ Inter-University Accelerator Centre, Aruna Asaf Ali Marg, New Delhi-110067, India
}

(Received 30 November 2008; published 23 April 2009)

\begin{abstract}
A superconducting linear accelerator based on niobium quarter wave resonators has recently become operational to boost the energy of the heavy ion beams available from the existing 15 UD (unit doubled) Pelletron accelerator. The niobium resonators typically performed at an accelerating field of 3-6 MV/m at 6 watts of input power in the test cryostat. When they were tested in the linac cryostat, the accelerating fields were drastically reduced and a number of other problems were also encountered. At present, all the problems have been diagnosed and solved. Many design modifications, e.g., in power coupler, mechanical tuner, helium cooling system, etc. were incorporated to solve the problems. A novel method of vibration damping was also implemented to reduce the effect of microphonics on the resonators. Finally, the accelerated beam through linac was delivered to conduct experiments.
\end{abstract}

DOI: 10.1103/PhysRevSTAB.12.040101

PACS numbers: 29.20.Ej, 85.25.- j

\section{INTRODUCTION}

Inter-University Accelerator Centre (IUAC) is equipped with a 15 UD (unit doubled) Pelletron [1] and routinely provides dc and pulsed ion beams in the energy range of a few tens to a few hundreds of $\mathrm{MeV}$ to perform experiments in the fields of nuclear physics, materials science, atomic physics, biophysics, etc. With only the Pelletron accelerator in operation, the ion energy to exceed the Coulomb barrier for a symmetric beam-target system $(\sim 5 \mathrm{MeV}$ per nucleon) is limited up to mass of $40 \mathrm{amu}$. To increase this limit to mass $\sim 100 \mathrm{amu}$, the construction of a superconducting (SC) linear accelerator (linac) based on niobium quarter wave resonators (QWR) was taken up. Design and fabrication of the prototype and the first dozen resonators were carried out successfully in collaboration with Argonne National Laboratory (ANL), USA [2,3]. Cryostats and cryogenic distribution lines were fabricated indigenously and have been operational for the past few years [4]. The rf amplifiers, resonator controller, and all other associated electronics were developed in-house and have been working successfully $[5,6]$. The complete linac will consist of five cryostats, the first one is the superbuncher (SB) cryostat housing a single QWR, the next three linac cryomodules house eight QWRs each, and the last cryostat has two QWRs used as rebuncher/debuncher (Fig. 1). At present, the SB cryostat, a single linac module, and the rebuncher $(\mathrm{RB})$ are operational. The other two linac cryostats will be operational from the middle of 2009. During the initial beam acceleration [7] with the resonators in SB, linac, and RB cryostats, a number of problems were encountered and many modifications in the design of the power coupler, mechanical tuner, cooling mechanism of resonators etc. were carried out. In addition, a new method of vibration damping was developed which enabled operation of the resonators at reduced power levels [8]. The details of the SC linac of IUAC, its different operational problems, their solutions, and the results of the beam acceleration are presented in this paper.

\section{NIOBIUM QUARTER WAVE RESONATOR}

For the past few decades, most of the superconducting heavy ion linac were either built with quarter wave [9-13] or half wave resonant structure $[14,15]$. The accelerating structure of the superconducting linac of IUAC was chosen to be a niobium QWR for its better mechanical stability and broad velocity acceptance so that a single structure could be used for the entire linac. The complete linac will consist of 27 QWRs, out of which the first 12 were fabricated in collaboration with ANL and the rest of the resonators are being fabricated in-house.

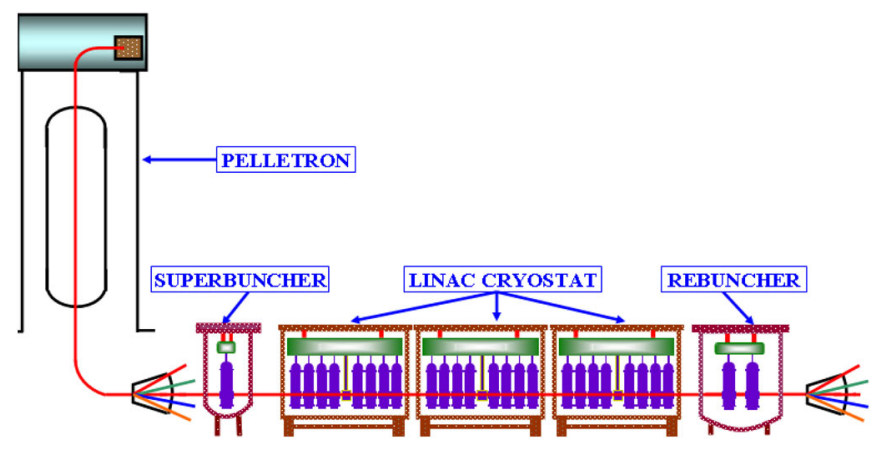

FIG. 1. (Color) The schematic of the Pelletron and the superconducting linear accelerator of IUAC. 
The accelerating field of a resonator is defined as [9]

$$
E_{a}=\frac{V_{o}}{b} T(\beta),
$$

where

$$
\begin{aligned}
V_{o} & =\text { peak rf voltage at the open end of the resonator } \\
2 b & =\text { inner diameter of the resonator } \\
T(\beta) & =\text { transit time factor, taken to be unity for measurement of accelerating field }
\end{aligned}
$$

The resonator parameters referenced at an accelerating electric field of $1 \mathrm{MV} / \mathrm{m}$ are given in the following:

$\begin{array}{ll}\text { Resonant frequency } & 97 \mathrm{MHz} \\ \text { Synchronous velocity } & 0.08 \mathrm{c} \\ \text { Drift tube voltage } & 85 \mathrm{KV} \\ \text { Energy content } & 110 \mathrm{~mJ} \\ \text { Peak magnetic field } & 106 \mathrm{G} \\ \text { Peak electric field } & 3.9 \mathrm{MV} / \mathrm{m} \\ \text { Geometric factor } & 17.3 \\ \text { Active length } & 15.9 \mathrm{~cm}\end{array}$

The cross-sectional view, the photograph of the resonators and the mechanical tuner are shown in Figs. 2-4, respectively. The resonator is formed of bulk niobium and is jacketed by another enclosure made out of stainless

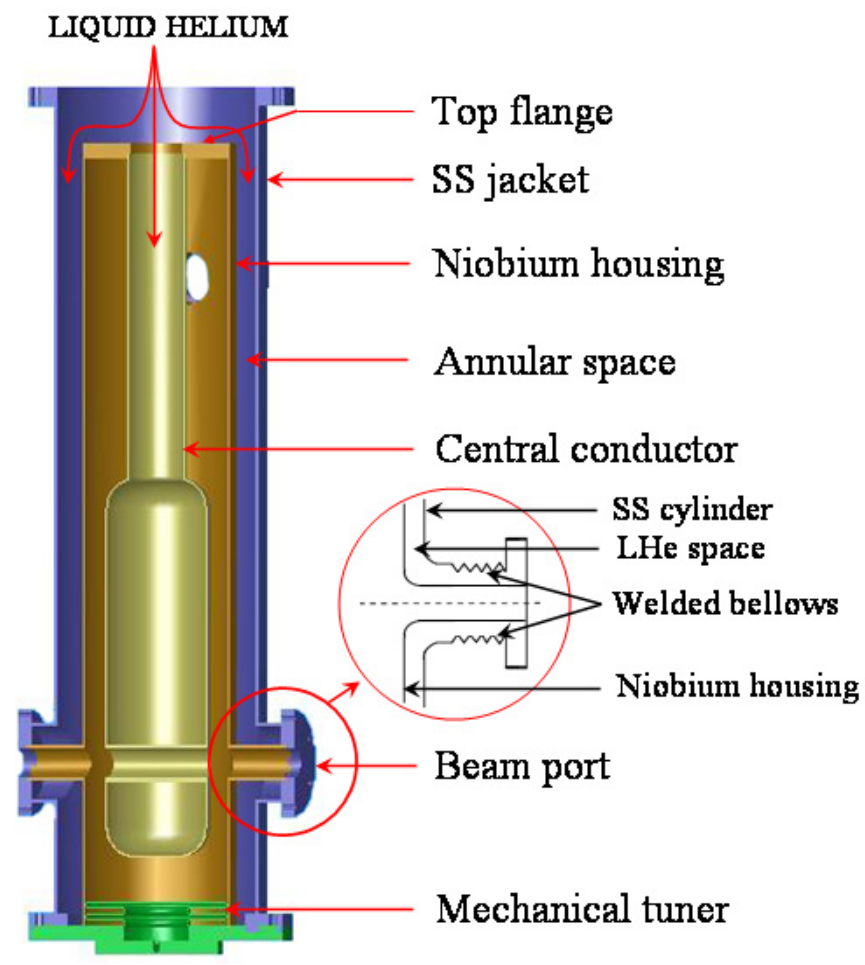

FIG. 2. (Color) The cross-sectional view of the resonator with the SS jacket, the beam ports, and the dismountable mechanical tuner. steel. Liquid helium ( $\mathrm{LHe}$ ) is poured into the central conductor as well as inside the annular space between the two concentric cylinders. The places where the outer stainless steel jacket joins the niobium resonator (to provide the power coupling ports, pickup ports, and open end flanges at the tuner end), a transition flange made by explosively bonded niobium-SS material is used to provide the welding transition between niobium and SS. Details of the design are presented elsewhere [2,3].

The rf power is coupled into the resonator by an inductively coupled loop installed on a movable rack and pinion arrangement. This is a standard method of coupling low level rf power (a few to hundreds of watts) into the superconducting resonators used to accelerate heavy ions $[10,16]$. A small amount of power from the resonator is picked up by another inductive pickup loop. Mechanical tuning of the resonator is accomplished by a flexible niobium bellows connected at the high voltage end of the resonator. Helium gas is used to flex niobium bellows (Fig. 4), changing the overall capacitance and hence the resonance frequency of the resonator can be varied. This frequency tuning, occurring in a time scale of a second or

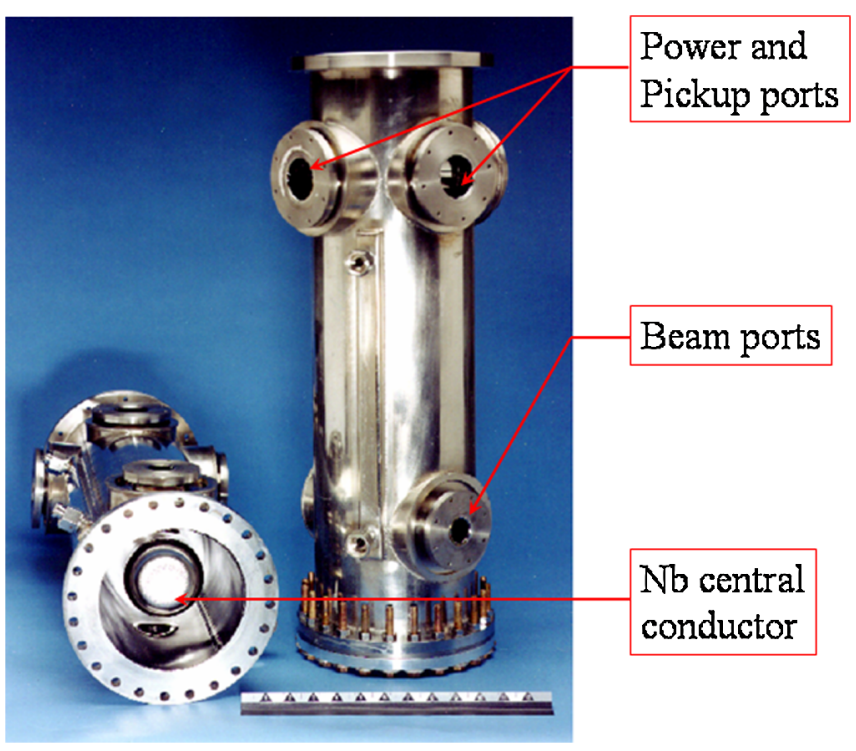

FIG. 3. (Color) Two complete quarter wave resonators with the SS jacket. 


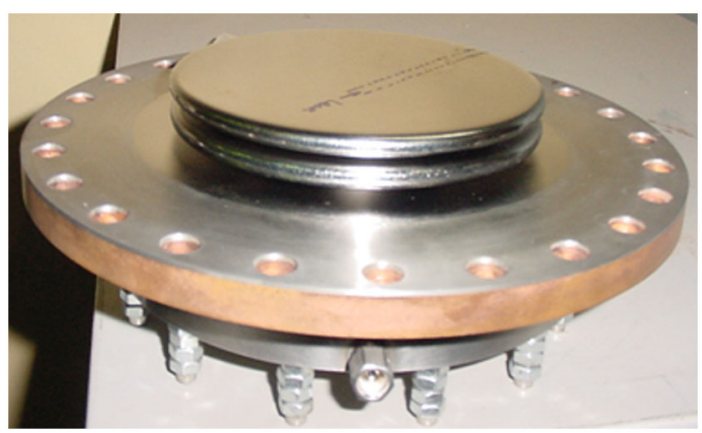

FIG. 4. (Color) The niobium bellows used as the mechanical tuner of the resonator.

more, takes care of slow drifts of frequency due to helium pressure fluctuation, etc. The frequency tuning, required at a faster time scale (tens of microseconds to few hundreds of milliseconds) is taken care of by the dynamic phase control mechanism. This mechanism [17], being simple and reliable, is used successfully in the control of the superconducting linac in various laboratories [13,18]. Both the slow and fast tuning [5] of the resonance frequency are an integral part of the resonator controller working in a closed loop control mechanism following the frequency of the master oscillator.

\section{PERFORMANCE TESTS OF THE RESONATORS IN TEST AND LINAC CRYOSTATS}

Prior to loading the resonators in the linac cryostat, their performances were checked in the test cryostat. The resonators were found to undergo heavy multipacting which usually takes 18-20 hours to clean up. This is a consequence of the large coaxial area between the lower part of the inner conductor and the outer housing wall where two points multipactoring can take place. The gap voltage at which two point multipacting of order $n$ can occur can be written as [19]

$$
V_{n}=E_{o} d=\frac{d^{2} \omega^{2} m}{(2 n-1) \pi e}
$$

and the nonrelativistic impact energy at

$$
t=\frac{(2 n-1) \pi}{\omega} \text { is } K_{n}=\frac{2 e^{2} V_{n}^{2}}{m \omega^{2} d^{2}}
$$

where

$$
\begin{aligned}
E_{0} & =\text { peak electric field } \\
d & =\text { gap length in meter } \\
\omega & =\text { resonance frequency }(2 \pi f) \\
m & =\text { mass of an electron } \\
e & =\text { electronic charge }
\end{aligned}
$$

For the distance of $3.35 \mathrm{~cm}$ between these two surfaces (the lower part of the central conductor and the housing of the QWR), the average electron energy resonant with the rf field at $97 \mathrm{MHz}$ turns out to be $\sim 480 \mathrm{eV}$. At this electron energy, the secondary electron emission coefficient for niobium is greater than 1 . The rf pulse conditioning is carried out for a period of 1-2 hours on each QWR to reduce the effect of field emission. The field measurements were performed at critically coupled condition by using the movable power coupler. The design goal of the accelerating field for our resonators is $4 \mathrm{MV} / \mathrm{m}$ at 6 watts of input power dissipated in liquid helium (LHe). During the past few years, numerous cold tests were performed to measure the performance of the niobium resonators. The plot of quality factor $(Q)$ vs accelerating fields of a few resonators is shown in Fig. 5.

In most of the performance tests in the test cryostat, the average accelerating fields achieved for the resonators were about $4 \mathrm{MV} / \mathrm{m}$ at 6 watts of input power as shown in Fig. 6. The resonators were phase and amplitude locked using the dynamic phase control method. The frequency jitter in the resonator was controlled by a combination of two different tuning devices - the slow and the fast tuner.

As shown in Fig. 6, the average performance for the resonators at 6 watts of power dissipated in $\mathrm{LHe}$ in the linac cryostat was found to be much lower than the average performance in the test cryostat. One of the possible reasons behind the field degradation was thought to be due to the inefficient cooling in some of the high current regions of the resonator.

The microphonics vibration in the linac cryostat was found to be higher than that in the test cryostat. Thus, a much higher level of forward power was required to phase lock the resonators in the linac cryostat. The high rf power resulted in several operational problems like melting of insulation of the rf power cable, excessive heating of the drive coupler leading to other associated problems, and increased cryogenic loss. The problems encountered in the

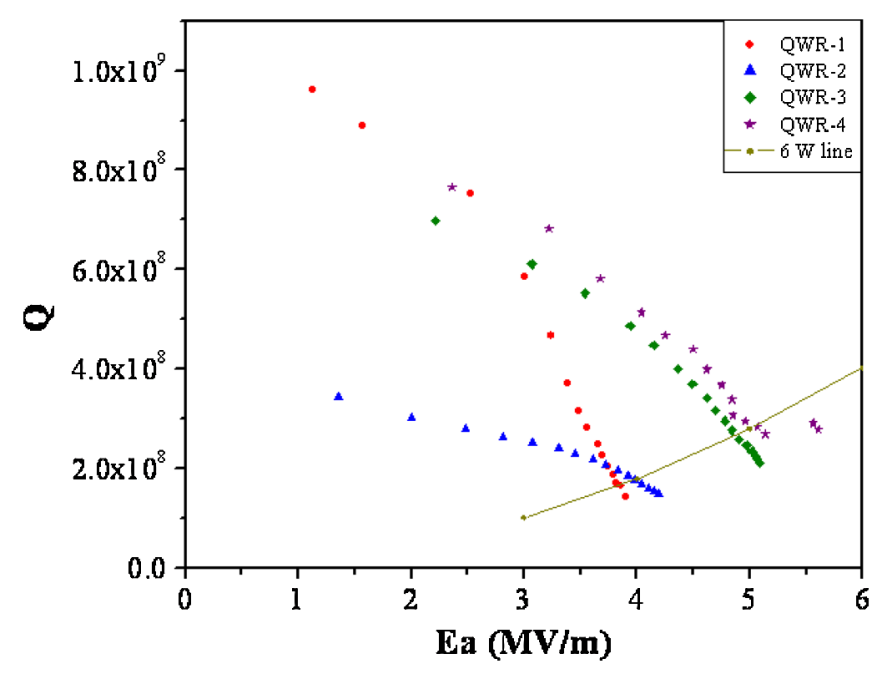

FIG. 5. (Color) Quality factor versus accelerating fields for four QWRs. 


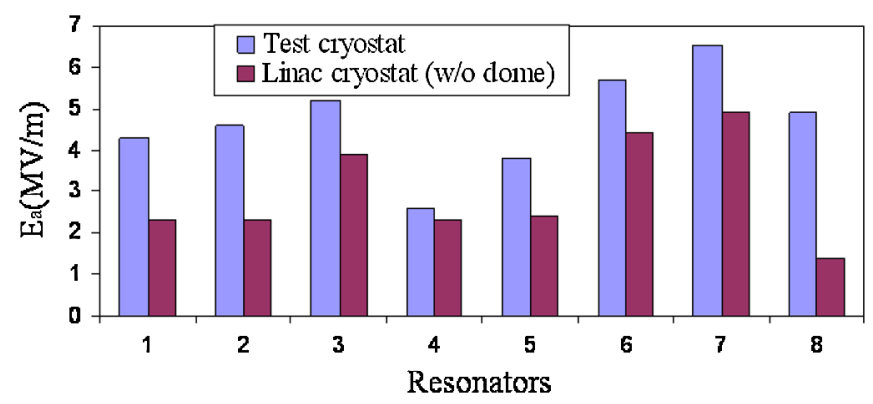

FIG. 6. (Color) Accelerating field performance of the resonators in the test cryostat and the linac cryostat prior to installation of the dome structure.

linac cryostat and their solutions are discussed in the following subsections.

\section{A. Improvement of cooling of the QWR}

The rf current reaches a maximum value in the region of the top flange of the resonator when it is powered. So inefficient cooling of this flange would have a deleterious effect on the resonator performance. During the initial tests with the resonators in the linac cryostat, a drop in the accelerating field was observed after the resonator had been powered for about half an hour. A possible cause for this could be trapping of helium gas bubbles between the flat niobium and the SS surface of the helium enclosure as shown in Fig. 7(a). Subsequently, the flat SS flange was replaced with a SS hemispherical dome, as shown in Fig. 7(b), and the drop in resonator fields after prolonged rf power was not observed (Fig. 8).
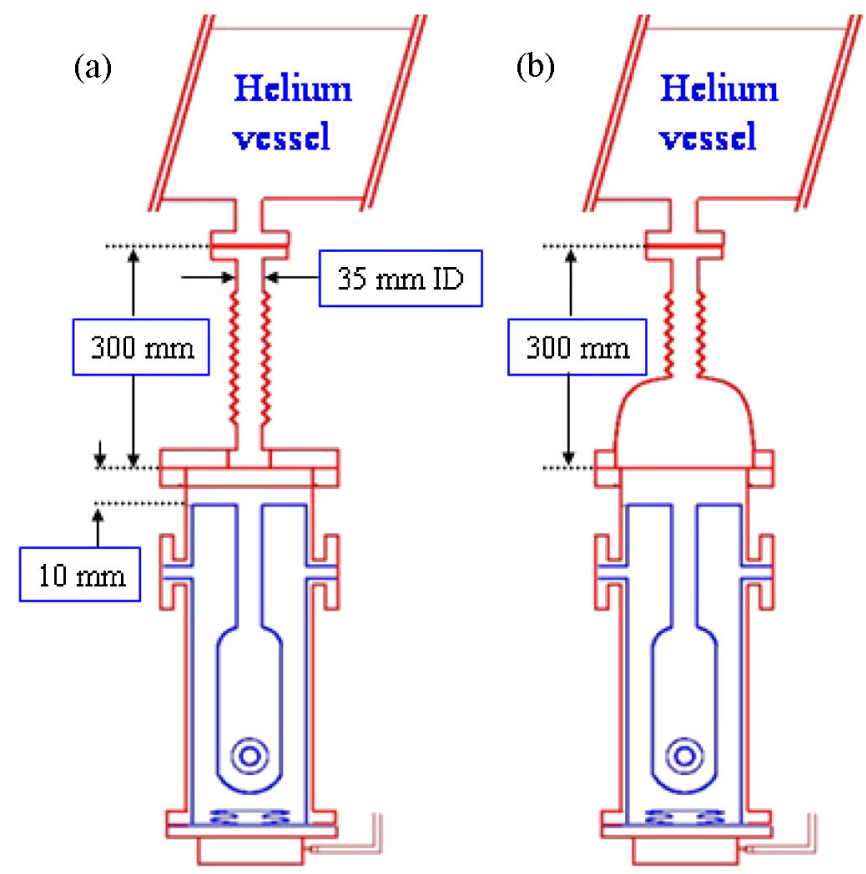

FIG. 7. (Color) The (a) old and (b) new configuration of the cooling schematic of QWR in the linac cryostat.

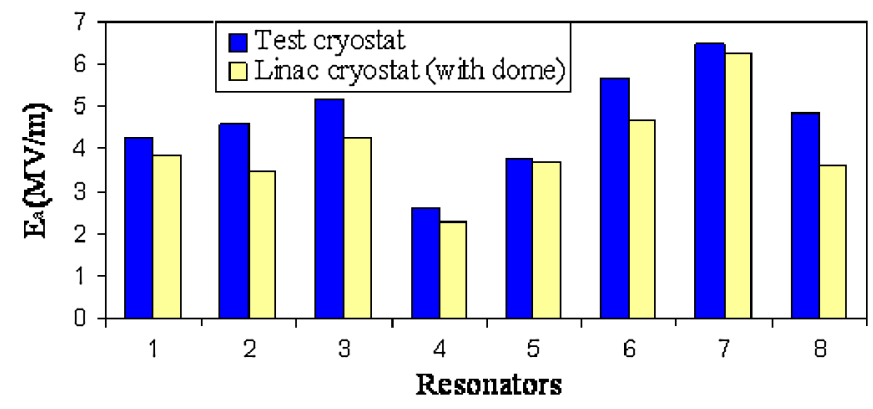

FIG. 8. (Color) Accelerating field performance of the resonators in the test cryostat and the linac cryostat after installation of the dome structure.

\section{B. Damping of microphonics of the resonator}

During the operation of QWRs in the linac cryostat, a typical quality factor of $\sim 2.0 \times 10^{8}$ corresponding to a field of $3-5 \mathrm{MV} / \mathrm{m}$ at 6 watts of $\mathrm{rf}$ power loss in the resonator is usually obtained with the modified dome structure mentioned in the previous paragraph. To achieve a stable operation of the superconducting resonators, dynamic feedback control of amplitude and phase [5] was adopted. A frequency window of $\pm 50 \mathrm{~Hz}(\Delta f)$ was required for locking the resonators due to the presence of microphonic noises. To obtain this large bandwidth, resonators were strongly overcoupled corresponding to a reduced $Q$ value $\sim 1 \times 10^{6}$ with a corresponding coupling coefficient value of $\beta \sim 200$. At this condition, the power required to lock the resonator was found to be $\sim 300$ watts. For resonators having a central conductor with uniform diameter, a mechanical damping mechanism had been tried out successfully elsewhere [20]. However, a similar damping mechanism could not be adopted since the central conductor of IUAC QWR has two different diameters. The reduction of microphonics was accomplished by an innovative and inexpensive method. Ordinary polished stainless steel balls of diameter $4.0 \mathrm{~mm}$ were inserted at the end of the helium side of the drift tube [Fig. 9]. The dynamic friction between the balls and the niobium surface acted to damp the oscillation reducing the amplitude of the vibration of the mechanical mode substantially. A reduction of $\sim 50 \%$ or more in the overall frequency excursion $(\Delta f)$ and the forward power requirement of the resonator were measured with SS balls in repeated experiments using different QWRs. During the last off-line performance test in the linac cryostat, it was observed that, with the new damping mechanism, a forward power of less than 100 watts was adequate for every resonator to lock at its maximum achievable field $(3-5 \mathrm{MV} / \mathrm{m})$ at $\sim 6$ watts of power going into LHe [8].

\section{Redesign of the power coupler}

The earlier operation of the power coupler, with forward power of 150-300 watts from the rf amplifier, resulted in the coupler getting heated to a temperature of $150^{\circ} \mathrm{C}$ or 


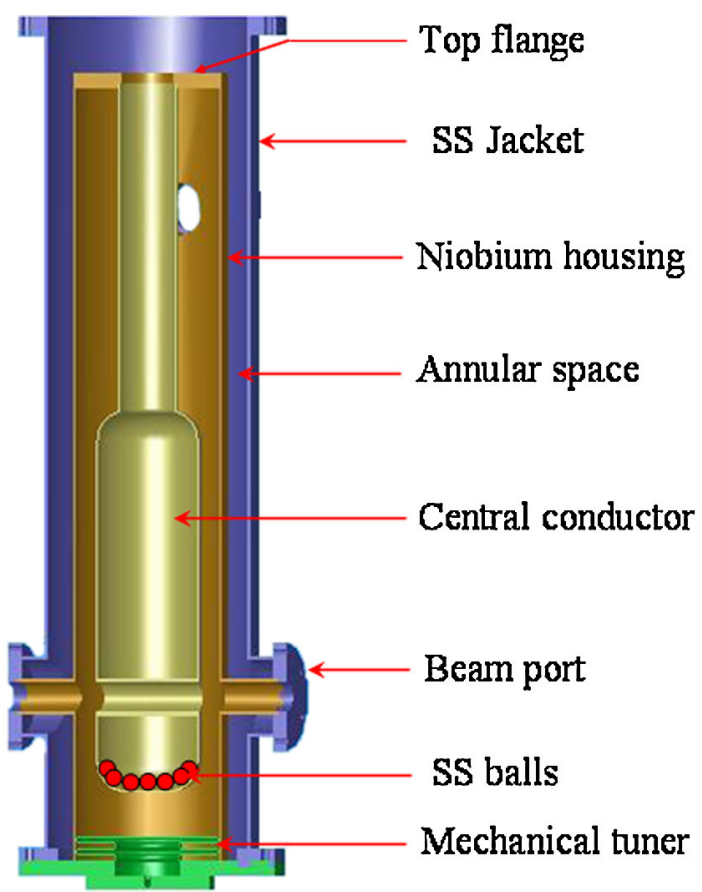

FIG. 9. (Color) Cross-sectional view of a resonator along with a few SS balls.

higher. This high temperature caused local vacuum deterioration, leading to multipactor discharge in the coupler resulting in further increase of local heating and subsequent zinc deposition from the brass material of the rack (of the rack and pinion arrangement) of the drive coupler (Fig. 10), onto the niobium surface of the resonator. To eliminate any possibility of metal contamination on the niobium surface during the operation of the resonators in the future, even with the reduced power of 100 watts, the

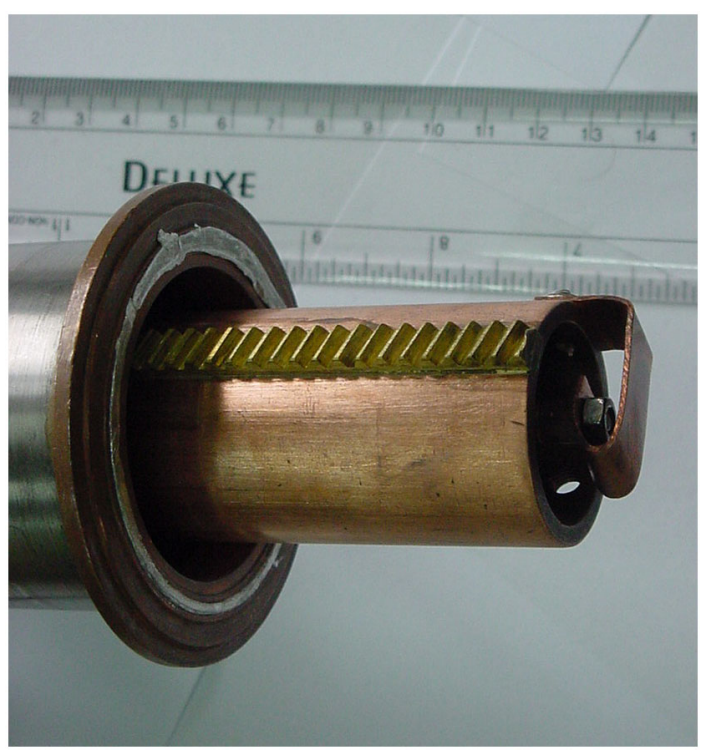

FIG. 10. (Color) The old power coupler.

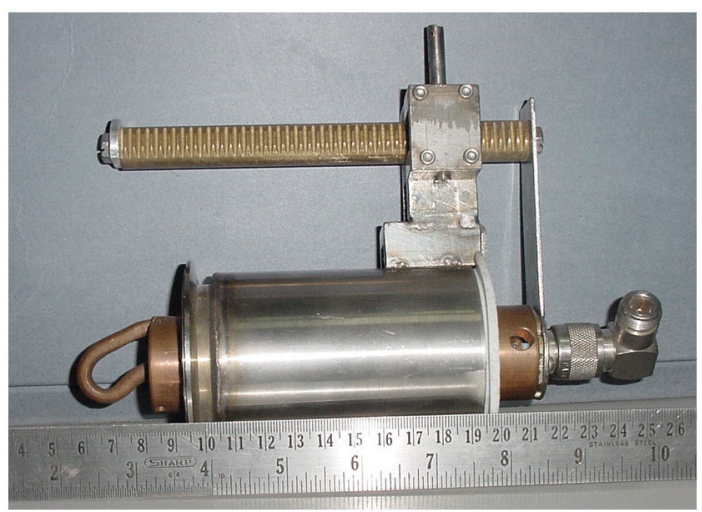

FIG. 11. (Color) The new power coupler.

design of the drive coupler was modified (Fig. 11) by using the rack outside the drive so that it does not enter into the resonator interior. During several off-line tests of the resonators with the new drive couplers with forward rf power up to 100 watts, no metal deposit was observed on the niobium surface or on the inside portion of the drive coupler.

\section{Modifications of the transition flange assembly}

Since the liquid helium enclosure was made of SS, explosively bonded $\mathrm{Nb}$-SS transition flanges were used to join the coupling/beam ports and the open end flange at the tuner side of the resonator. Edge welded SS bellows were used in all the coupling/beam ports of the first 12 resonators (shown in Fig. 12) to accommodate the differential contraction between niobium and SS at $4.2 \mathrm{~K}$.

After a few thermal cycles, the thin edge welded SS bellows started leaking in the pressurized condition caus-

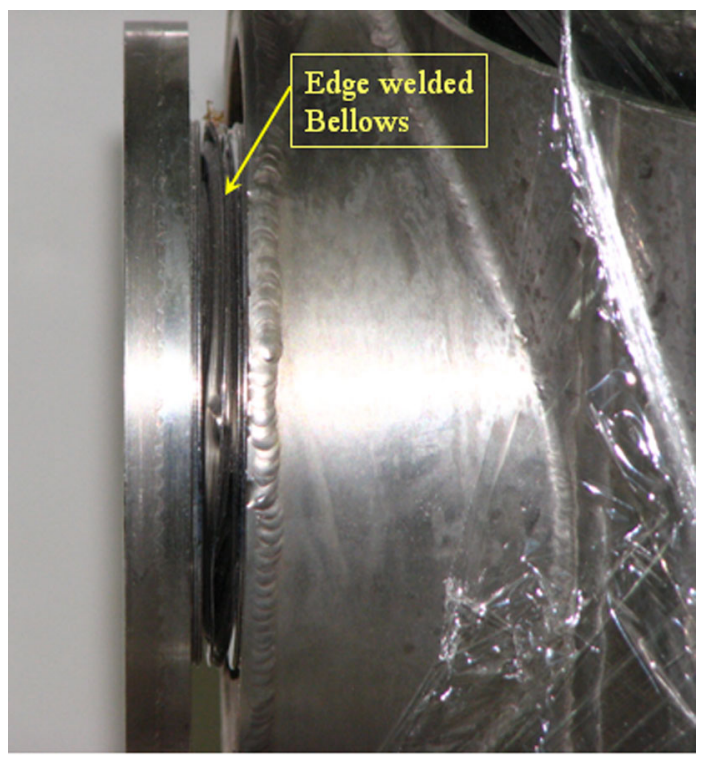

FIG. 12. (Color) Edge welded bellows to join the niobium-SS transition flanges with the SS jacket. 


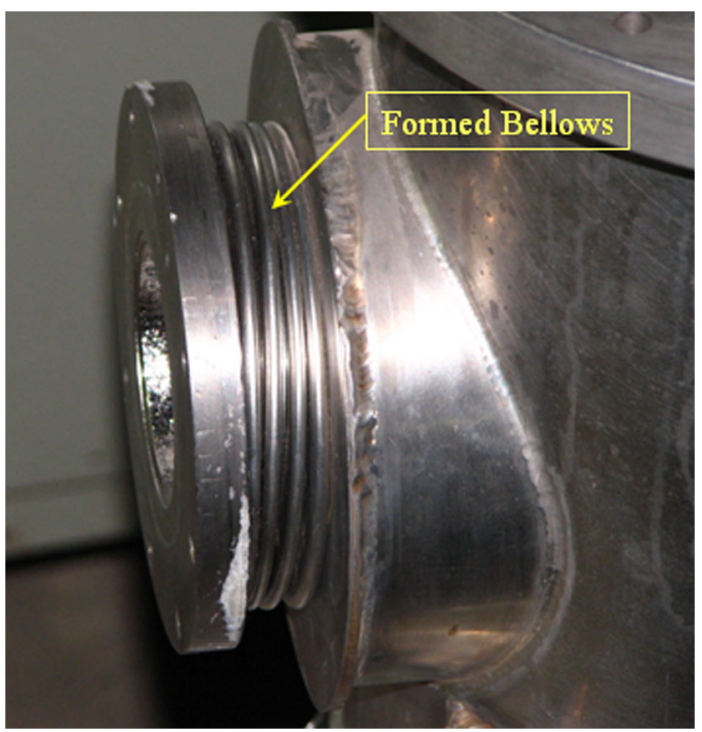

FIG. 13. (Color) Newly replaced formed bellows to join the niobium-SS transition flanges with the SS jacket.

ing deterioration of the cryostat vacuum. The thin edge welded bellows were subsequently replaced by thicker formed SS bellows (shown in Fig. 13) [21] and with this modification, the leaks from the bellows have been eliminated.

\section{E. Modifications of the fixture of the mechanical tuner}

The mechanical tuner is a device consisting of an edge welded niobium bellows using $0.8 \mathrm{~mm} \mathrm{Nb}$ sheets as shown in Fig. 4. These bellows were operated using helium gas directly to flex it and, hence, a vacuum seal with an indium gasket separating the cryostat vacuum from the helium gas was required. On repeated thermal cycles, two types of leaks were observed. One was due to helium gas leaking through the indium seal and the other was leaks of helium through the weld cracks of the niobium bellows. To eliminate these problems, the fixture of the mechanical tuner was modified in such a way that helium gas flexes another

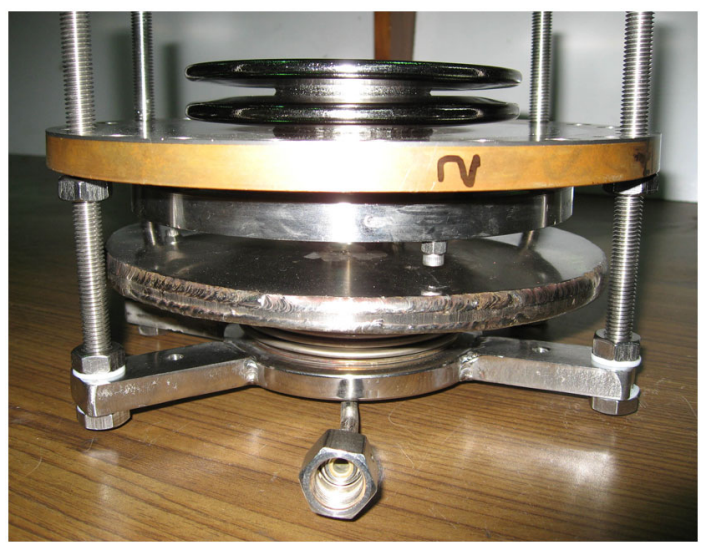

FIG. 14. (Color) The mechanical tuner with the SS bellows and shaft to pull/push the niobium convolutions. additional SS bellows which pushes or pulls the niobium convolution by a SS shaft to tune the resonator's frequency (Fig. 14). In the new design, helium gas does not fill the niobium bellows eliminating the need for the vacuum seal.

\section{SUCCESSFUL BEAM ACCELERATION}

After incorporating all the modifications, on-line beam acceleration with the resonators of the first linac module was performed. To measure the energy and the time pulse length of the beam, two scattering chambers were placed one before and another after the linac cryostat. Silicon surface barrier detectors were used to measure the time and energy of the scattered particles from a thin gold foil. During beam acceleration, ${ }^{28} \mathrm{Si}^{+10}$ beam of $130 \mathrm{MeV}$ from the Pelletron accelerator was prebunched to $\sim 1.5 \mathrm{nsec}$ by a multiharmonic buncher. The unwanted beam currents between the successive beam bunches were removed by a high energy sweeper [22] located in the post-tandem section. With careful optimization of the phase and amplitude of the resonator acting as a superbuncher, the bunched beam was further compressed to $\sim 250 \mathrm{psec}$ at the entrance of the linac cryostat (Fig. 15).

The beam with 250 ps pulse length was then injected into the linac module containing seven resonators (the eighth one was removed from the cryostat due to a leak problem) and a total energy gain of about $28 \mathrm{MeV}$ obtained from the seven resonators was measured by another thick surface barrier detector $(300 \mu \mathrm{m})$ at the exit of the linac (Fig. 16).

The accelerated beam was transported up to the rebuncher (RB), located about 14 meters down the line from the first linac cryostat. By optimizing the reference phase of a single resonator of the RB cryostat and then by changing the amplitude of the accelerating field, the time width of the beam bunch measured at the user's scattering chamber was compressed from $1.1 \mathrm{~ns}$ to $\sim 400$ ps (Fig. 17).

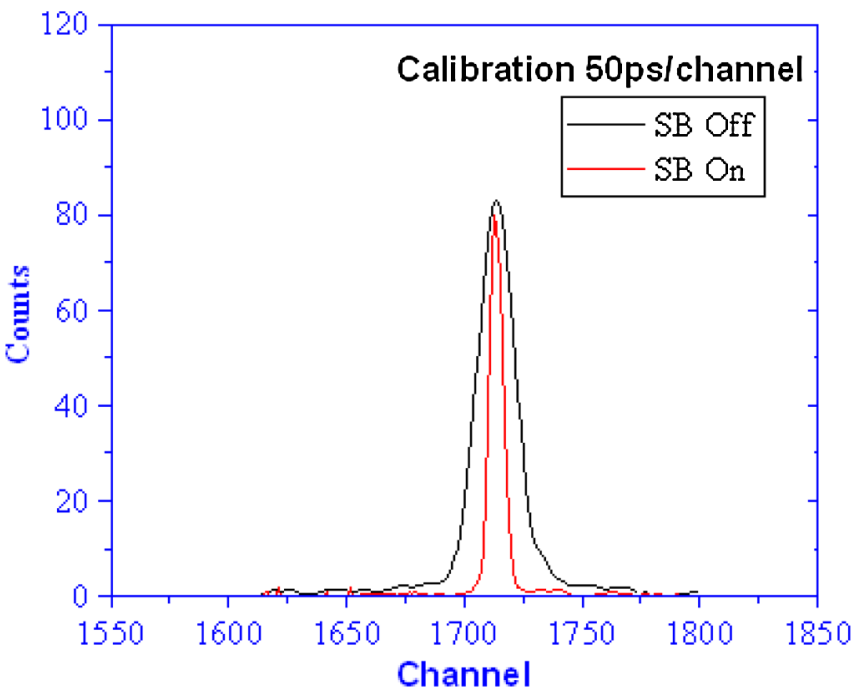

FIG. 15. (Color) Pulse length of ${ }^{28} \mathrm{Si}$ beam at the linac entrance. 


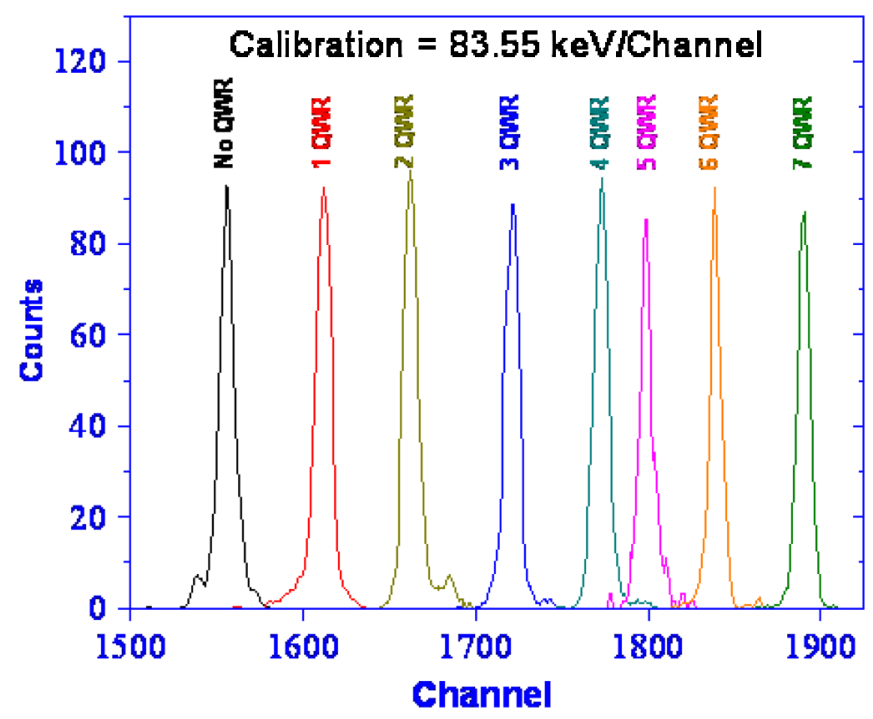

FIG. 16. (Color) Energy gain after each resonator.

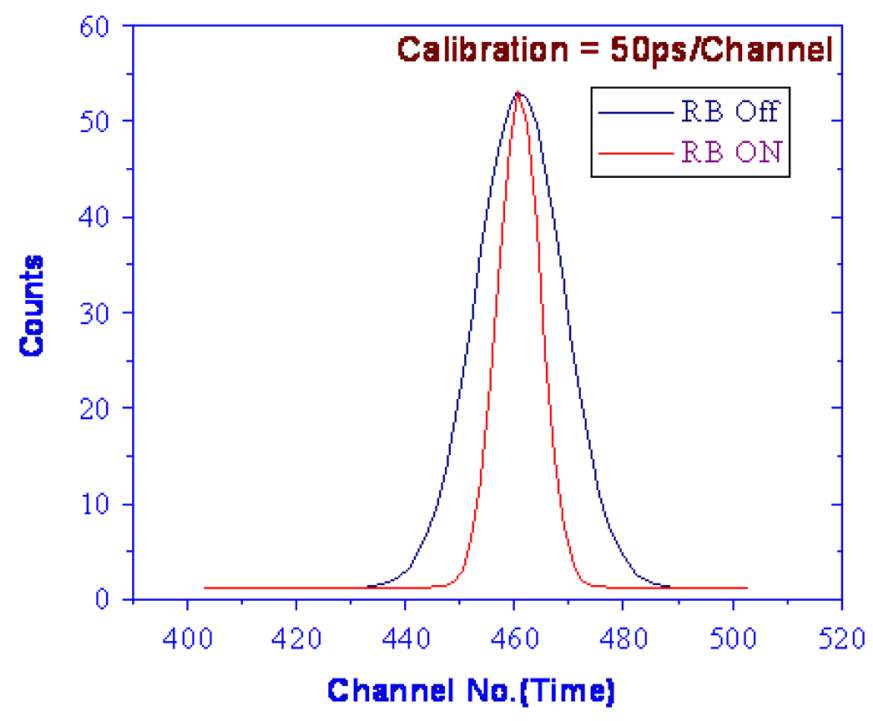

FIG. 17. (Color) Pulse length of ${ }^{28} \mathrm{Si}$ beam at the target location.

In a subsequent experiment, $100 \mathrm{MeV},{ }^{16} \mathrm{O}^{+8}$ beam from the Pelletron, prebunched by the multiharmonic buncher and low energy chopper, to a pulse length of $\sim 1.0$ ns was injected into SB. The resonator in SB produced a pulse length of $\sim 160 \mathrm{ps}$ at the linac entrance and after acceleration by seven resonators in the linac, a total energy of $120 \mathrm{MeV}$ was obtained at the exit of linac. This beam was then rebunched by a QWR in RB to a time width of $\sim 500 \mathrm{ps}$ and was delivered at the experimental chamber to conduct experiments.

\section{CONCLUSION}

Prebunched beams from the Pelletron were rebunched using the superbuncher and accelerated using QWRs in the first linac module and then rebunched again using the rebuncher successfully. The accelerated beam was delivered for conducting nuclear physics experiments. The new designs of the power coupler, slow tuner, the dome structure to improve cooling of the resonator, and the damping mechanism had eliminated problems encountered earlier. All the modifications were incorporated in the present module and will be incorporated in the next two linac modules.

\section{ACKNOWLEDGMENTS}

The authors would like to thank the Pelletron group members, especially Mr. R. Joshi and Mr. A. Sarkar, for their help during the time of beam acceleration through the linac. Help received from Mr. Akhil Jhingan is also acknowledged.

[1] D. Kanjilal et al., Nucl. Instrum. Methods Phys. Res., Sect. A 328, 97 (1993).

[2] K. W. Shepard, A. Roy, and P. N. Potukuchi, in Proceedings of the 17th Particle Accelerator Conference, Vancouver, BC, Canada, 1997 (IEEE, New York, 1997), p. 3072.

[3] P. N. Potukuchi, S. Ghosh, and K.W. Shepard, in Proceedings of the 18th Particle Accelerator Conference, New York, 1999 (IEEE, New York, 1999), p. 952.

[4] T.S. Datta et al., in Proceedings of the Asian Particle Conference (RRCAT, Indore, India, 2007), p. 397.

[5] G. Joshi, C. I. Sujo, B. K. Sahu, A. Pandey, A. Kumar B. P., and J. Karande, in Proceedings of the Ninth International Conference of Heavy Ion Accelerator Technology, New Delhi, India, 2002 [Pramana J. Phys. 59, 1035 (2002)].

[6] B. K. Sahu, A. Pandey, G. Joshi, C. I. Sujo, A. Kumar B. P., and A. Roy, in Proceedings of the Indian Particle Accelerator Conference, InPAC 2005, p. 223.

[7] S. Ghosh et al., in Proceedings of the Indian Particle Accelerator Conference, 2005, Kolkata, India, p. 48.

[8] S. Ghosh, P. Patra, B. K. Sahu, A. Rai, G. K. Chaudhari, A. Pandey, D. Kanjilal, and A. Roy, Phys. Rev. ST Accel. Beams 10, 042002 (2007).

[9] I. Benzvi and J. M. Brennan, Nucl. Instrum. Methods 212, 73 (1983).

[10] A. M. Porcellato, G. Bisoffi, S. Gustaffsson, L. Boscagli, D. Carlucci, F. Chiurlotto, M. Morvillo, and F. Stivanello, Nucl. Instrum. Methods Phys. Res., Sect. A 382, 121 (1996).

[11] S. Takeuchi, T. Ishii, and H. Ikezoe, Nucl. Instrum. Methods Phys. Res., Sect. A 281, 426 (1989).

[12] K. W. Shepard, P. K. Markovich, G. P. Zinkann, B. Clifft, and R. Benaroya, in Proceedings of the 1989 Particle Accelerator Conference, IEEE, 1989, p. 974.

[13] R.E. Laxdal et al., in Proceedings of the 20th Particle Accelerator Conference, Portland, OR, 2003 (IEEE, New York, 2003).

[14] K. W. Shepard, C. H. Sheibelhut, R. Benaroya, and L. M. Bollinger, IEEE Trans. Nucl. Sci. 24, 1147 (1977).

[15] J. R. Delayen and J. E. Mercereau, Nucl. Instrum. Methods Phys. Res., Sect. A 257, 71 (1987). 
[16] R. Poirier, K. Fong, P. Harmer, R. Laxdal, A. Mitra, I. Sekatchev, B. Waraich, and V. Zvyagintsev, in Proceedings of the LINAC 2004, Lubeck, Germany.

[17] I. Benzvi, M. Birk, C. Broude, G. Gitliz, M. Sidi, J. S. Sokolowski, and J. M. Brennan, Nucl. Instrum. Methods Phys. Res., Sect. A 245, 1 (1986).

[18] G. Fortuna et al., Nucl. Instrum. Methods Phys. Res., Sect. A 328, 236 (1993).

[19] H. Padamsee, J. Knobloch, and T. Hays, $R F$ Superconductivity for Accelerators (John Wiley and Sons, Inc., New York, 1988), p. 191.
[20] A. Facco, V. Zviagintsev, and B. Cheremushkinskaya, Proceedings of the Particle Accelerator Conference, Vancouver, BC, Canada, 1997 (Ref. [2]), p. 3084.

[21] A. Roy, in Proceedings of the 21st Particle Accelerator Conference, Knoxville, 2005 (IEEE, Piscataway, NJ, 2005), pp. 625-628.

[22] S. Ghosh, A. Sarkar, S. S. K. Sonti, S. Rao, V. V. Sivakumar, A. Rai, P. Barua, S. K. Dutta, R. K. Bhowmik, and A. Roy, Rev. Sci. Instrum. 77, 076103 (2006). 